\title{
3 Research Soure \\ ZBTB38 in Porcine Alveolar Macrophages Positively Regulates the Proliferation of Japanese Encephalitis Virus
}

\section{Yi Zheng}

Sichuan Agricultural University

\section{Yu-Yong Zhou}

University of Copenhagen Faculty of Health and Medical Sciences: Kobenhavns Universitet

Sundhedsvidenskabelige Fakultet

\section{Chun-Xia Chai}

Inner Mongolia University School of Life Science

\section{San-Jie Cao}

Sichuan Agricultural University

Qi-Gui Yan

Sichuan Agricultural University

\section{Xiao-Bo Huang}

Sichuan Agricultural University

\section{Yi-Ping Wen}

Sichuan Agricultural University

\section{Qin Zhao}

Sichuan Agricultural University

\section{Sen-Yan Du}

Sichuan Agricultural University

Rui Wu ( $\square$ wurui1977@163.com )

Sichuan Agricultural University https://orcid.org/0000-0001-7791-4167

\section{Research Article}

Keywords: ZBTB38, Japanese encephalitis virus, zinc finger protein, CRISPR/Cas9, Flavivirus

Posted Date: September 15th, 2021

DOl: https://doi.org/10.21203/rs.3.rs-856427/v1

License: (c) (1) This work is licensed under a Creative Commons Attribution 4.0 International License. Read Full License 


\section{Abstract}

Background Japanese encephalitis (JE) is an important zoonotic disease caused by Japanese encephalitis virus (JEV), and pigs are intermediate host of this disease. Previous studies have confirmed that JEV can proliferate in the respiratory tract of mice and spread through it. Therefore, this study aimed to screen the proteins interacting with JEV on porcine alveolar macrophage cell and verify its role in the proliferation of JEV.

Methods and results Porcine alveolar macrophages cell line 3D4/21 were infected with JEV, and obvious cytopathic effect (CPE) was observed. Zinc finger and BTB domain containing 38 (ZBTB38) was screened out as an interacting protein using co-immunoprecipitation assay and validated through knockout and overexpression of ZBTB38 in 3D4/21 cells. The results demonstrated that loss of ZBTB38 function basically had no effect on the attachment and entry processes of JEV, while the transcription level of JEV envelope gene, the expression level of NS3 protein and the number of virions were all significantly down-regulated in the subsequent infection stage.

Conclusion Overall, one core conclusion was drawn in this paper that ZBTB38 promotes the proliferation of JEV especially in the middle and late stages of infection. This study provides new information for understanding the pathogenic mechanism of JEV, especially the respiratory transmission caused by JEV infection.

\section{Introduction}

JEV is a single-stranded positive-sense RNA virus with envelope. It belongs to Flavivirus of Flaviviridae, and there are serological cross-reactions between viruses of this genus [9]. The genome of JEV has one open reading frame, from $5^{\prime}$ to $3^{\prime}$ end, which encodes three structural proteins (capsid (C), membrane (M), envelope (E)) and seven non-structural proteins (NS1, NS2A, NS2B, NS3, NS4A, NS4B, NS5) [21]. JEV has a spherical appearance with genome, capsid, and envelope from the inside to the outside. The structure of envelope is a phospholipid bilayer with several membrane proteins and glycoprotein fibers on it [3]. E protein as the main structural protein of JEV can bind to the receptor on the host cell membrane and mediate the fusion of the viral envelope and cell membrane [20]. Therefore, E protein plays a vital role in the entry process. In addition, E protein contains antigenic determinants and hemagglutinin which can directly affect the pathogenicity, cell tropism, hemagglutination and immunogenicity of JEV [13]. It is known that the non-structural proteins of JEV are closely related to the viral replication. Previous study showed that blocking the expression of NS3 and NS4A can significantly inhibit the replication of JEV both in Baby Hamster Kidney (BHK)-21 cells and mice [12]. Besides, the combination of NS5 protein and host cytokines can also effectively promote the replication of virus [22].

Flaviviruses have two main modes of transmission: mosquito-borne and tick-borne. Tick-borne flaviviruses are composed of a single serum complex, while mosquito-borne flaviviruses include JEV, 4 
serotypes of dengue virus (DENV), yellow fever virus (YFV) and others. DENV and YFV are transmitted by Aedes aegypti [5], and Culex mosquitoes is the vector of JEV. Aside from these traditional methods of transmission, many flaviviruses can also be transmitted without vectors. It was reported that DENV, YFV, West Nile virus (WNV) and Zika virus (ZIKV) can also spread vertically $[1,6-8,11,16]$. Besides, airborne transmission, blood transfusion and direct contact have also been reported in ZIKV and JEV infection [4, $14,15,19]$. Similarly, we previously investigated the proliferation of JEV through intranasal infection and showed that JEV can replicate in the lungs and spread through the respiratory tract of mice [2]. However, the underlying molecular mechanism of respiratory infections is unknown yet. In this study, the interacting protein ZBTB38 was screened out using co-immunoprecipitation assay. We aimed at knockout and overexpression ZBTB38 to study its role during different stages of JEV infection.

\section{Materials And Methods Virus and cells}

Genotype I JEV SCYA201201 strain (GenBank accession no. KM658163) was isolated from brain tissues of aborted piglets in China [24]. It was passaged in BHK-21 cells (ATCC), and virus titer was determined by plaque assay. BHK-21 cells were maintained as described previously [25]. Porcine alveolar macrophages cell line 3D4/21 (ATCC) and ZBTB38 knockout 3D4/21 cells (KO.ZBTB38 cells, generated from 3D4/21 cells as parental cells) were maintained in Roswell Park Memorial Institute (RPMI) 1640 Medium (Gibco, Grand Island, NY, USA) supplemented with $10 \%$ fetal bovine serum (FBS; Hyclone, South Logan, UT, USA), $100 \mu \mathrm{g} / \mathrm{ml}$ streptomycin and $100 \mathrm{U} / \mathrm{ml}$ penicillin in a $5 \% \mathrm{CO}_{2}$ incubator at $37^{\circ} \mathrm{C}$.

\section{Plasmids and antibodies}

The full-length ZBTB38 gene (NCBI accession no. NM_001195373.1) was amplified from 3D4/21 cells by PCR. The pEGFP-ZBTB38 plasmid was constructed by inserting the full-length PCR amplified ZBTB38 gene into the PEGFP-C3 vector which was digested with Xho I and Sal I. The primers used for plasmids construction and quantitative real-time RT-PCR in this study are listed in Table 1. Sequences of all the recombinant plasmids were verified by sanger dideoxy sequencing. High-immunized serum from mouse against JEV and mouse polyclonal antibody against JEV NS3 protein was generated in our laboratory [23]. The mouse polyclonal anti-ZBTB38 antibody was purchased from Thermo Fisher Scientific (Waltham, MA, USA) and the mouse monoclonal anti- $\beta$-actin antibody was supplied from ABclonal Technology (Wuhan, China). 
Table 1

Primer pairs used for plasmid construction and quantitative real-time RT-PCR.

\begin{tabular}{|ll|}
\hline Primer name & Sequence $\left(\mathbf{5}^{\prime} \boldsymbol{\rightarrow} \mathbf{3}^{\prime}\right)$ \\
\hline ZBTB38-F & tcagatctcgagctcaagcttGCCACCATGTCCCTTTCCAGGGACCTCA \\
\hline ZBTB38-R & ttatctagatccggtggatcCAAGGACATTATCAGCAAGGGTTT \\
\hline JEV-E-F & CAGTGGAGCCACTTGGGTG \\
\hline JEV-E-R & TTGTGAGCTTCTCCTGTCG \\
\hline$\beta$-actin-F & CTTCCTGGGCATGGAGTCC \\
\hline$\beta$-actin-R & TTGTGAGCTTCTCCTGTCG \\
\hline $\begin{array}{l}\text { Notes: F for forward primer, R for reverse primer. Lowercase letters are part of homologous } \\
\text { recombination. }\end{array}$ \\
\hline
\end{tabular}

\section{Virus infection}

Monolayers of cells were incubated with JEV at $37^{\circ} \mathrm{C}$ in a $5 \% \mathrm{CO}_{2}$ atmosphere for $1.5 \mathrm{~h}$. Unbound virus was removed by washing with serum-free medium. Then cells were maintained in medium with $2 \% \mathrm{FBS}$ at $37^{\circ} \mathrm{C}$ in a $5 \% \mathrm{CO}_{2}$ atmosphere for indicated time. The mock-infected cells were prepared using the same procedures without virus.

\section{Co-immunoprecipitation assay}

The membrane protein of 3D4/21 cells was extracted by Membrane Protein Extraction Kit (Bestbio Science, Shanghai, China) after JEV infection, and the protein was quantified using the BCA Protein Assay Kit (Sangon Biotech, Shanghai, China). Protein A magnetic beads were incubated with the highimmunized serum from mouse against JEV overnight at $4{ }^{\circ} \mathrm{C}$ by gentle shaking. The membrane protein was then added and incubated overnight at $4{ }^{\circ} \mathrm{C}$. JEV was added subsequently and the magnetic beads were washed three or more times with wash buffer. The bound proteins were eluted by boiling with SDSPAGE loading buffer for $5 \mathrm{~min}$ and then subjected to SDS-PAGE. LC-MS/MS analysis (Sangon Biotech) was carried out on specific protein bands, and the results were screened in protein database for pigs (Sus scrofa) (https://www.ncbi.nlm.nih.gov/).

\section{Western blotting}

Total cell lysates were prepared using Radio-Immunoprecipitation Assay Buffer (Sigma-Aldrich, St. Louis, MO, USA) containing protease inhibitors (Roche, Bassel, Switzerland). Protein concentrations were determined using the BCA Protein Assay Kit (Sangon Biotech). Proteins were separated by SDS-PAGE and then transferred to a nitrocellulose filter membrane (Bio-Rad, Hercules, CA, USA) using a Trans-Blot SemiDry Transfer apparatus (Bio-Rad). $\beta$-actin was used as an internal control. Blots were probed with the relevant antibodies, and proteins were detected using enhanced chemiluminescence reagent (Bio-Rad). 


\section{Construction of ZBTB38 knockout 3D4/21 cell line by CRISPR/Cas9 system}

The online CRISPR tool (http://crispr.mit.edu/) was used to design specific sgRNA for ZBTB38. To reduce off-target effects, we designed two pairs of sgRNA (sgRNA1: 5'- CACCGCTTAAATGAGCAGCGCATC-3' and 5'-AAACGGATGCGCTGCTCATTTAAGC-3'; sgRNA2: 5'-CACCGATCCAGTTCCAGGACGTGGC-3' and 5'AAACGCCACGTCCTGGAACTGGATC-3'). The two oligos of sgRNA were cloned into pSpCas9n(BB)-2APuro (PX459) plasmid (Addgene). The recombinant plasmids were transfected into 3D4/21 cells. Puromycin $(5.5 \mu \mathrm{g} / \mathrm{mL})$ was added for selecting positive transfected cells. Cell clones with ZBTB38 knockout were confirmed by DNA sequencing and immunofluorescence assay.

\section{Transfection assay}

Transfections were done in 60-mm dishes using Lipofectamine 3000 (Invitrogen, Carlsbad, CA, USA) in both 3D4/21 cells and KO.ZBTB38 cells. All procedures were performed according to the manufacturer's recommendations.

\section{Viral attachment and entry assay}

For viral attachment assay, KO.ZBTB38 cells were seeded in 6-well plate, and 3D4/21 cells were used as control. Each group has three replicates. The supernatants were removed when the cells reach $90 \%$ confluent. After one time wash with PBS, the cells were infected with JEV at multiplicity of infection (MOI) of 10 . After $1 \mathrm{~h}$ incubation at $4^{\circ} \mathrm{C}$, the cells were washed twice with cold PBS. The cells were spined down by centrifugation, and then total RNA was extracted. Quantitative RT-PCR was used to detect the genomic level of viral attachment. For viral entry assay, cells were incubated at $4^{\circ} \mathrm{C}$ for $1 \mathrm{~h}$ after JEV infection, and then transferred to $37^{\circ} \mathrm{C}$ for 1 more hour. After removal of supernatants, cells were washed one time with cold PBS, and then added $400 \mu \mathrm{L}$ proteinase $\mathrm{K}(1 \mathrm{mg} / \mathrm{mL})$ per well. After 45 min incubation at $4^{\circ} \mathrm{C}$, the supernatants were removed and the cells were washed three times with cold PBS. Total RNA was then extracted and quantitative RT-PCR was used to detect the genomic level of viral entry.

\section{Immunofluorescence assay}

KO.ZBTB38 cells were seeded on cover slips in 12-well plate, and 3D4/21 cells were used as control. Monolayers of cells were washed with PBS and fixed with $4 \%$ paraformaldehyde for $12 \mathrm{~h}$ at $4{ }^{\circ} \mathrm{C}$. And then cells were washed with PBS three times and blocked with $5 \%$ Bovine serum albumin (BSA) in PBS for $12 \mathrm{~h}$. Anti-ZBTB38 antibody in blocking solution was incubated with the cells for $12 \mathrm{~h}$ at $4{ }^{\circ} \mathrm{C}$. Cells were washed three times and incubated with anti-mouse FITC IgG for $1 \mathrm{~h}$ at $37^{\circ} \mathrm{C}$. After final wash, DAPI staining was performed for $10 \mathrm{~min}$. Cover slips were mounted on glass slide, and images were captured using Olympus BX51 fluorescence microscope.

\section{RNA extraction and quantitative real-time RT-PCR}

Cells were treated or infected as indicated. Then, total RNA was extracted using TRIzol Reagent (Sangon Biotech). Reverse transcription was performed using PrimeScript RT Reagent Kit with gDNA Eraser 
(TaKaRa Bio, Tokyo, Japan). Synthetic cDNA was analysed for quantitative real-time PCR, using SYBR Premix Ex Taq II (TliRNaseH Plus) (TaKaRa Bio) in a CFX96 Real-Time PCR Detection System (Bio-Rad). Relative mRNA values were calculated using the $2^{-\Delta \Delta C T}$ method. $\beta$-actin was used as an internal control in each sample and showed as fold change by normalizing to the mock-control.

\section{Plaque assay}

The supernatants from JEV infected cultures were serially diluted and inoculated onto the monolayer of BHK-21 cells which were seeded in six-well plates. After $1.5 \mathrm{~h}$ incubation at $37^{\circ} \mathrm{C}$, the cells were washed with serum-free Dulbecco's modified Eagle's medium (DMEM; Gibco) and cultured in DMEM containing $2 \%$ FBS and $1.5 \%$ sodium carboxymethyl cellulose for 4 days. The cells were then stained with crystal violet and fixed with formaldehyde for $30 \mathrm{~min}$ at room temperature. Visible plaques were counted, and the virus titer was calculated. All data are expressed as means of triplicate samples.

\section{Quantification and statistical analysis}

All statistical analyses and calculations were performed using GraphPad Prism (Version 6, La Jolla). All data are expressed as means \pm standard deviation (SD) as indicated. Student's unpaired t test was used to estimate the statistical significance between two groups, whereas ANOVA was used to compare the means among three or more groups. The $p$-value less than 0.05 was considered statistically significant. Statistical significance is indicated as * $(p<0.05),{ }^{\star *}(p<0.01),{ }^{\star \star *}(p<0.001)$ and ns (not significant).

\section{Results}

\section{ZBTB38 in 3D4/21 cells was screened as an interacting protein of JEV}

3D4/21 cells were infected with JEV (MOI 1), and obvious CPE was observed $48 \mathrm{~h}$ post-infection (Fig. 1A), which indicated that JEV could infect 3D4/21 cells and cause CPE. After the co-immunoprecipitation assay, SDS-PAGE analysis revealed the cellular factors that interact with JEV (Fig. 1B). There was a specific band with 70 to $100 \mathrm{kDa}$ in size in co-immunoprecipitation group (lane 1) which did not exist in virus control group (lane 2) and antibody control group (lane 3), while it can be observed in the membrane protein control group (lane 4). The specific band was cut off for LC-MS/MS analysis, and the results are listed in Table 2, including HSPA8, ZBTB38, VIM and HSP70.2. 
Table 2

The results of LC-MS/MS.

\begin{tabular}{|lllll|}
\hline No. & Protein Name & Gene & ACCESSION & Score \\
\hline 1 & heat shock 70kDa protein 8 [Sus scrofa] & HSPA8 & NP_001230836.1 & 73 \\
\hline 2 & $\begin{array}{l}\text { Zinc finger and BTB domain containing 38 [Sus } \\
\text { scrofa] }\end{array}$ & ZBTB38 & ADC38889.1 & 69 \\
\hline 3 & vimentin, partial [Sus scrofa domesticus] & VIM & ABA39527.1 & 65 \\
\hline 4 & heat shock protein 70.2 [Sus scrofa] & HSP70.2 & AAR30953.1 & 62 \\
\hline
\end{tabular}

Lack of ZBTB38 does not affect the attachment and entry of JEV

Using CRISPR/Cas9 system, we generated 3D4/21 cells deficient in ZBTB38 expression. The results of immunofluorescence assay showed that KO.ZBTB38 cells had almost no specific fluorescence compared with 3D4/21 cells (Fig. 2A). Relative quantitative RT-PCR was performed to evaluate the effects of ZBTB38 deficiency in the attachment and entry processes of JEV (Fig. 2B and C). In the viral attachment and entry stage, there was no significant difference between KO.ZBTB38 group and control group. This suggests that ZBTB38 has no effects on the attachment and entry processes of JEV.

\section{Knockout ZBTB38 weakens the replication and attenuates the virulence of JEV}

The results after 36 and $48 \mathrm{~h}$ JEV infection showed that the level of JEV mRNA in 3D4/21 cells was upregulated with the progress of infection, but this phenomenon was not observed in KO.ZBTB38 cells. Meanwhile, the level of JEV mRNA were both significantly lower than that in the control group (Fig. 3A), demonstrated that knocking out ZBTB38 gene significantly inhibited the transcription of JEV. After $48 \mathrm{~h}$ JEV infection, total protein of cells was extracted. As shown in (Fig. 3B), after ZBTB38 knocking out, the NS3 protein was significantly down-regulated indicated that ZBTB38 gene-deficient cells significantly inhibited the expression of JEV protein. After $72 \mathrm{~h}$ of JEV infection, the supernatants were collected for plaque assay. Compared with the control group, the virus titer of JEV in KO.ZBTB38 cells was significantly down-regulated (Fig. 3C) indicated that loss of ZBTB38 gene inhibited the release of JEV virions.

\section{Trans-complementation and overexpression of ZBTB38 promote JEV Infection}

As shown in (Fig. 4A), after trans-complementing ZBTB38 gene, the mRNA level of JEV in KO.ZBTB38 cells was about 1.5 times higher than the control group. Taken together, ZBTB38 can promote the transcription of JEV genes. After $48 \mathrm{~h}$ JEV infection, total protein of cells was extracted. When ZBTB38 protein was overexpressed in 3D4/21 cells, NS3 protein expression was up-regulated compared with the 
control group indicated that ZBTB38 can promote the expression of JEV protein (Fig. 4B). However, compared with the KO.ZBTB38 cells, the up-regulation of NS3 protein level after trans-complementation was not significant. After $72 \mathrm{~h}$ JEV infection, the supernatants were collected for plaque assay. As shown in (Fig. 4C), the virus titer of KO.ZBTB38-ZBTB38 group was significantly up-regulated compared with the control group illustrated that ZBTB38 can promote the release of virions.

\section{Discussion}

Candidate proteins such as HSPA8, ZBTB38 and VIM with high scores were screened out after LC-MS/MS analysis. ZBTB38, a zinc finger protein with $\mathrm{C} 2 \mathrm{H} 2$ and $\mathrm{BTB}$ domains, aroused our interest. Some zinc finger proteins related to the proliferation of JEV have been confirmed such as MCPIP1, a zinc finger protein with a highly conserved $\mathrm{CCCH}$-type structure, which is an RNA-binding enzyme that can not only target viral RNA, but also effectively promote antiviral effects. Besides, overexpression of MCPIP1 can inhibit the replication of JEV and DENV [18]. Taken together, we speculated that ZBTB38 may also be involved in the proliferation of JEV. Searching the public database, ZBTB38 is a protein located in the nucleus. However, the LC-MS/MS analysis results showed that ZBTB38 was one of the candidate proteins interacting with JEV. The reason for this may be that the membrane proteins were not accurately extracted, and the protein samples used in the experiment were mixed with proteins from other parts of the cell. In view of the high score of ZBTB38, we continue to study it for the next step.

In order to reduce the off-target probability and improve the knockout efficiency, we designed two pairs of sgRNA. The survival rate of 3D4/21 cells in 1640 medium with different concentrations of puromycin was observed. The optimal screening concentration was $5.5 \mu \mathrm{g} / \mathrm{ml}$. Immunofluorescence assay results showed that the expression of ZBTB38 in KO.ZBTB38 cells was basically suppressed, but there was still some vague fluorescence can be observed, indicated that ZBTB38 was not completely knocked out. Further research should take isolation of monoclonal cell population into consideration for reducing the interference of normal cells on the experimental results.

After confirming that the loss of ZBTB38 function has no effect on the attachment and entry process of JEV, we compared the proliferation of JEV in KO.ZBTB38 cells and 3D4/21 cells. After KO.ZBTB38 cells were infected with JEV, the mRNA level of JEV was significantly reduced by about 9 times compared with the control group. In addition, the JEV mRNA was significantly increased after trans-complementing ZBTB38 and infected with JEV. It can be seen from the results that ZBTB38 positively regulates the level of JEV mRNA. The degree of difference in the trans-complementation experiment is not as great as the knockout experiment, which may be caused by experimental operation. It is consistent that the expression of JEV NS3 was significantly down-regulated after KO.ZBTB38 cells were infected with JEV and was significantly up-regulated after 3D4/21 cells overexpressed ZBTB38. However, in the transcomplementation experiment, the expression of NS3 was not significantly different from the control group. The above findings showed that knocking out ZBTB38 cannot completely block the proliferation of JEV indicated that ZBTB38 is not the single protein that regulates the proliferation of JEV, and further study is needed. 
Considering that ZBTB38 may have no effect on the packaging and release of virions when JEV infects the cells for $48 \mathrm{~h}$, the incubation time was extended to $72 \mathrm{~h}$ after infection, and supernatants were collected for plaque assay. Compared with the control group, the virus titer of the knockout group decreased significantly, and the titer increased in trans-complementation group. On this basis, we supposed that ZBTB38 also exerted an influence on the assembly and release of the virions. Through gene knockout, overexpression, and trans-complementation experiments, we verified the pivotal role of ZBTB38 in the proliferation of JEV. These results demonstrated that ZBTB38 positively regulates the proliferation of JEV.

ZBTB38, which is called CIBZ in humans (Homo sapiens), was found to be related to Casepase-3. Knocking out of CIBZ can induce apoptosis [17]. Until now, the effect of ZBTB38 on the proliferation of flavivirus is not clear. Among many proteins with zinc finger structures, in addition to the aforementioned MCPIP1 that can inhibit the replication of JEV, CCCH-type Zinc-finger Antiviral Protein (ZAP), as an endogenous antiviral factor, has also been reported to be able to specifically bind to viral RNA and prevent JEV infection. YFV, DENV and ZIKV, which are also flaviviruses, have been reported to have the ability of ZAP-resistant [10]. Therefore, it cannot be simply confirmed that zinc finger protein must have antiviral function. According to the results of this study, we believe that JEV is likely to have the ability to resist ZBTB38 and might be activated by ZBTB38. Although the effect of ZBTB38 on flavivirus has not been reported yet, based on the effects of other zinc finger proteins on the virus, it is plausible that this gene may also have a vital impact on JEV and other flavivirus.

\section{Declarations}

\section{Funding}

This work was supported by Sichuan Science and Technology Program in China (2020YFS0574) and National natural science foundation of China (3197190576). The funders had no role in study design, data collection and analysis, decision to publish, or preparation of the article.

\section{Conflict of interest}

None of the authors has any other financial or personal relationships that could inappropriately influence or bias the content of the paper.

\section{Ethical approval}

This article does not contain any studies with human participants or animals performed by any of the authors.

\section{Consent for publication}

Not applicable. 


\section{References}

1. Ary F, Crans WJ, Patricia B, Bruce W, Burkhalter KL, Godsey MS, Aspen SE, Nasci RS (2005) Detection of West Nile Viral RNA from an Overwintering Pool of Culex pipens pipiens (Diptera: Culicidae) in New Jersey, 2003. Journal of Medical Entomology:490

2. Chai C, Palinski R, Xu Y, Wang Q, Cao S, Geng Y, Zhao Q, Wen Y, Huang X, Yan Q, Ma X, Wen X, Huang $Y$, Han X, Ma W, Wu R (2019) Aerosol and Contact Transmission Following Intranasal Infection of Mice with Japanese Encephalitis Virus. Viruses 11

3. Chambers TJ, Hahn CS, Galler R, Rice CM (1990) Flavivirus genome organization, expression, and replication. Annu Rev Microbiol 44:649

4. Cheng VCC, Siddharth S, Shuk-Ching W, Wong SCY, Chan JFW, Yip CCY, Chi-Hung C, Au TWK, Yu-Yan H, Yau CSW (2018) Japanese Encephalitis Virus Transmitted Via Blood Transfusion, Hong Kong, China. Emerging Infectious Diseases 24:49-57

5. Cleland JB, Bradley B, Macdonald W (1919) Further Experiments in the Etiology of Dengue Fever. Epidemiology Infection 18:217-254

6. Colt S, Garcia-Casal MN, Peña-Rosas JP, Finkelstein JL, Mehta S (2017) Transmission of Zika virus through breast milk and other breastfeeding-related bodily-fluids: A systematic review. PLOS Neglected Tropical Diseases 11:e0005528

7. Foy BD, Kobylinski KC, Foy JLC, Blitvich BJ, Amelia TDR, Haddow AD, Lanciotti RS, Tesh RB (2011) Probable non-vector-borne transmission of Zika virus, Colorado, USA. Emerg Infect Dis 17:880-882

8. Günther J, Martínez-Muñoz JP, Pérez-Ishiwara DG, Salas-Benito J (2007) Evidence of vertical transmission of dengue virus in two endemic localities in the state of Oaxaca. Mexico Intervirology 50:347-352

9. Heinz FX, Stiasny K (2012) Flaviviruses and flavivirus vaccines. Vaccine 30:4301-4306

10. Hsin-Ping C, Han C, Chao-Fu Y, Yi-Ling L, Feng-Lan C, Hung-Chih K, Ren-Jye L, Yi-Ling L, Schoggins JW (2018) Inhibition of Japanese encephalitis virus infection by the host zinc-finger antiviral protein. Plos Pathogens 14:e1007166

11. Kuhn S, Twele-Montecinos L, Macdonald J, Webster P, Law B (2011) Case report: probable transmission of vaccine strain of yellow fever virus to an infant via breast milk. Can Med Assoc $J$ 183:E243-E245

12. Lei, Yuan, Rui, Wu H, Liu, Xintian, Wen X, Huang (2016) The NS3 and NS4A genes as the targets of RNA interference inhibit replication of Japanese encephalitis virus in vitro and in vivo. Gene 594:183-189

13. Luca VC, AbiMansour J, Nelson CA, Fremont DH (2011) Crystal Structure of the Japanese Encephalitis Virus Envelope Protein. J Virol 86:2337-2346

14. Lyons A, Huang Y-J, Park SL, Ayers V, Hettenbach S, Higgs S, Mcvey D, Noronha L, Hsu W-W, Vanlandingham D (2018) Shedding of Japanese Encephalitis Virus in Oral Fluid of Infected Swine. Vector-Borne Zoonotic Diseases 18:469-474 
15. Moreira J, Peixoto TM, Siqueira AM, Lamas CC (2017) Sexually acquired Zika virus: a systematic review. Clin Microbiol Infect 23:296-305

16. O'Leary DR, DVMa, Kuhn S, Kniss KL, Hinckley AF (2006) Birth Outcomes Following West Nile Virus Infection of Pregnant Women in the United States: 2003-2004. Pediatrics 117:537-545

17. Oikawa Y, Matsuda E, Nishii T, Ishida Y, Kawaichi M (2008) Down-regulation of CIBZ, a Novel Substrate of Caspase-3, Induces Apoptosis. J Biol Chem 283:14242-14247

18. Ren-Jye L, Hsu-Ling C, Shyr-Yi L, Bi-Lan C, Han-Pang Y, Wei-Chun T, Yi-Ling L (2013) MCPIP1 ribonuclease exhibits broad-spectrum antiviral effects through viral RNA binding and degradation. Nucleic Acids Res 41:3314-3326

19. Ricklin ME, García-Nicolás O, Brechbühl D, Python S, Zumkehr B, Nougairede A, Charrel RN, Posthaus H, Oevermann A, Summerfield A (2016) Vector-free transmission and persistence of Japanese encephalitis virus in pigs. Nat Commun 7:10832

20. Shukla J, Bhargava R, Dash PK, Parida M, Tripathi N, Rao PVL (2009) Cloning and expression of domain III of the envelope gene of Japanese encephalitis virus: evaluation for early clinical diagnosis by IgM ELISA. J Virol Methods 158:165-170

21. Villordo SM, Gamarnik AV (2009) Genome cyclization as strategy for flavivirus RNA replication. Virus Res 139:230-239

22. Wang B, Thurmond S, Hai R, Song J (2018) Structure and function of Zika virus NS5 protein: perspectives for drug design. Cellular Molecular Life Sciences 75:1-14

23. Xu Y, Wang Q, Wei B, Huang X, Wen Y, Yan Q, Ma X, Zhao Q, Cao S, Huang Y, Wen X, Han X, Bai Y, Wu R (2019) Enhanced Immune Responses Against Japanese Encephalitis Virus Infection Using Japanese Encephalitis Live-Attenuated Virus Adjuvanted with Montanide GEL 01 ST in Mice. Vector Borne Zoonotic Dis 19:835-843

24. Yuan L, Wu R, Liu H, Wen X, Huang X, Wen Y, Ma X, Yan Q, Huang Y, Zhao Q, Cao S (2016) Tissue tropism and molecular characterization of a Japanese encephalitis virus strain isolated from pigs in southwest China. Virus Res 215:55-64

25. Zhao Z, Wu G, Zhu X, Yan X, Dou Y, Li J, Zhu H, Zhang Q, Cai X (2012) RNA interference targeting virion core protein ORF095 inhibits Goatpox virus replication in Vero cells. Virology Journal 9:48

\section{Figures}


A

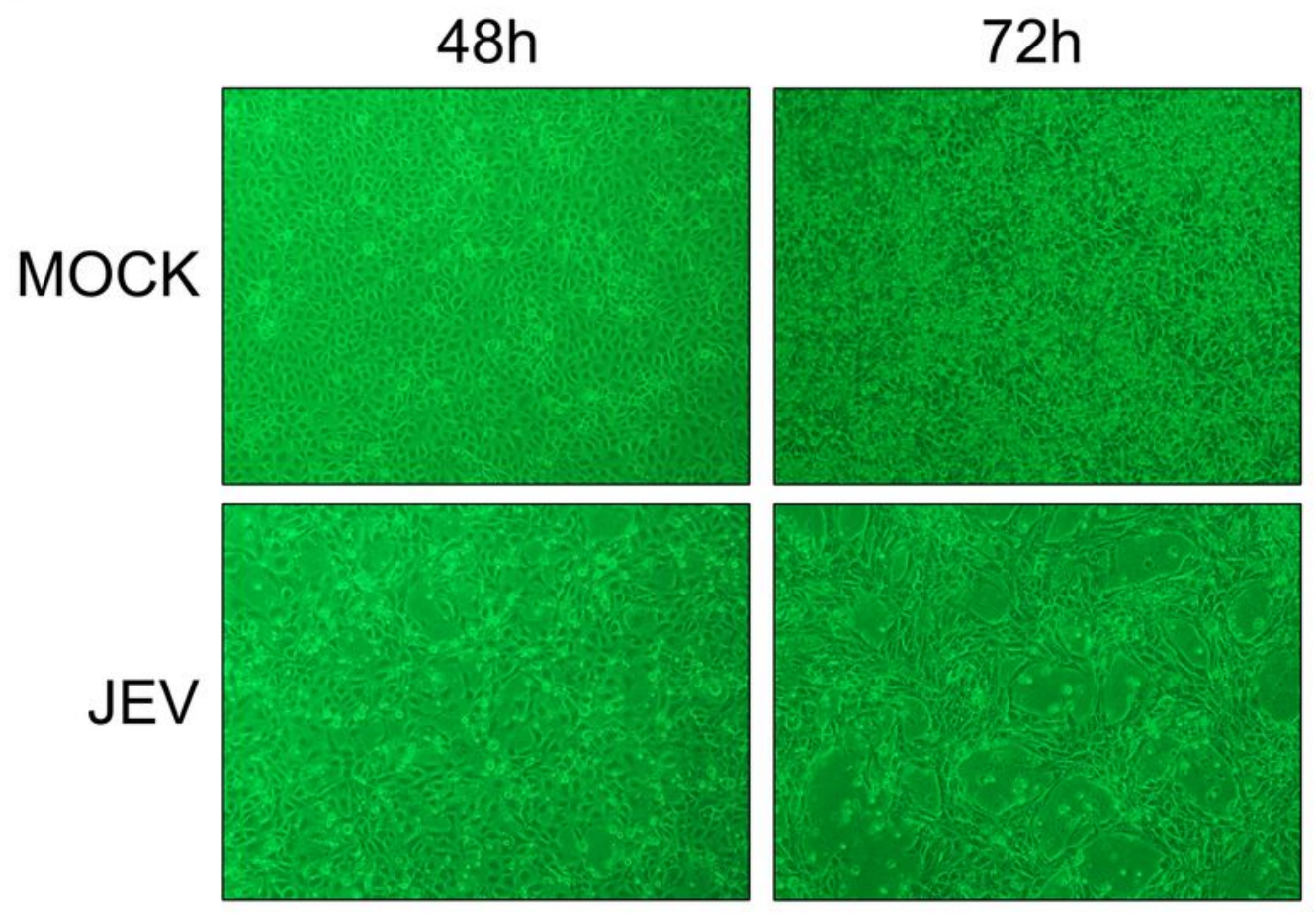

$\mathrm{B}$

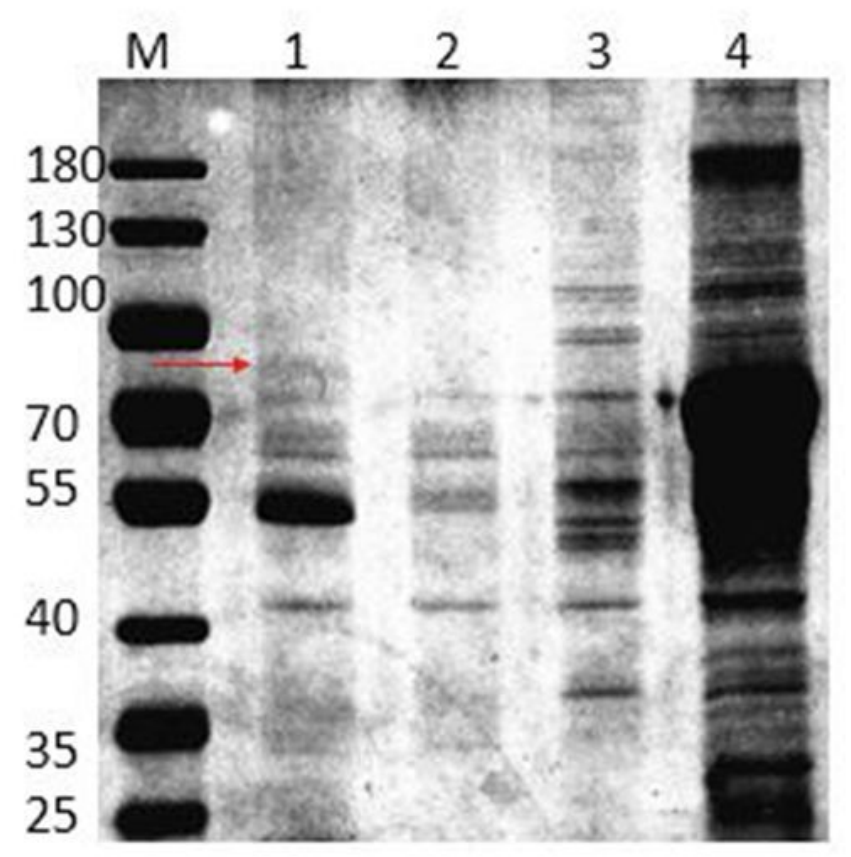

Figure 1

3D4/21 cells were susceptible to JEV and the interacting proteins were screened. A The infected 3D4/21 cells showed evident CPE (MOI 1, 48 and $72 \mathrm{~h}$ post-infection). B SDS-PAGE analysis of coimmunoprecipitation. Lane M: protein marker; lane 1: the product of co-immunoprecipitation group (with JEV, antibody and membrane protein); lane 2: the virus control group (with JEV only); lane 3: the antibody 
control group (with antibody only); lane 4: the membrane protein control group (with membrane protein only). Specific protein band was marked with red arrows

A

Anti-ZBTB38

$3 \mathrm{D} 4 / 21$
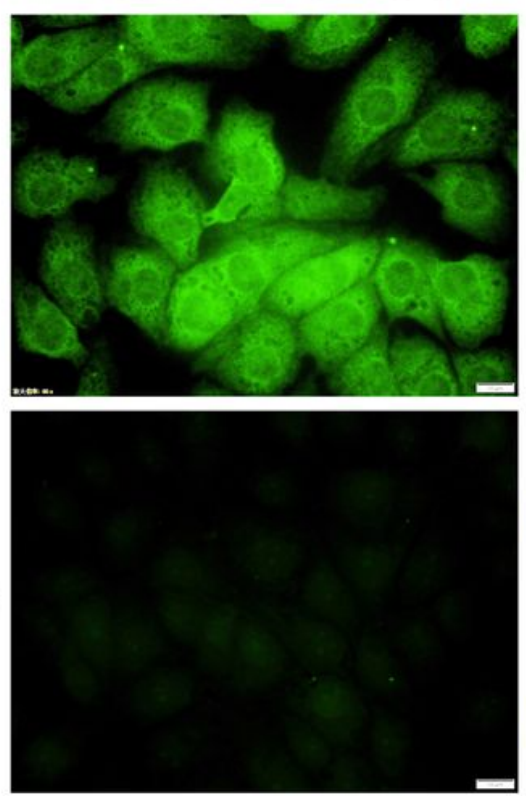

KO.ZBTB38

B

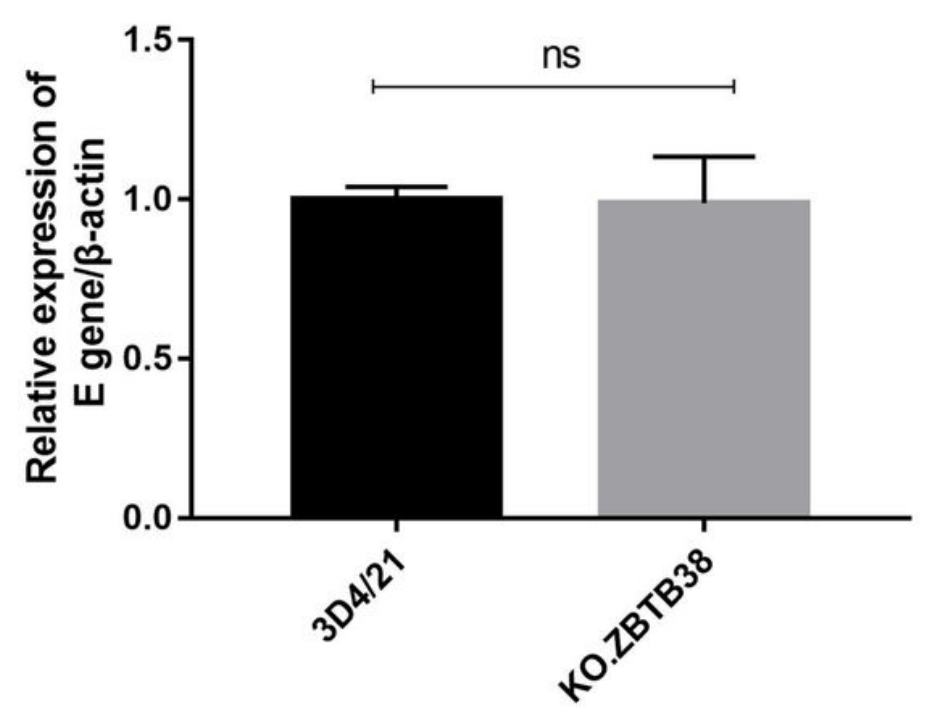

DAPI
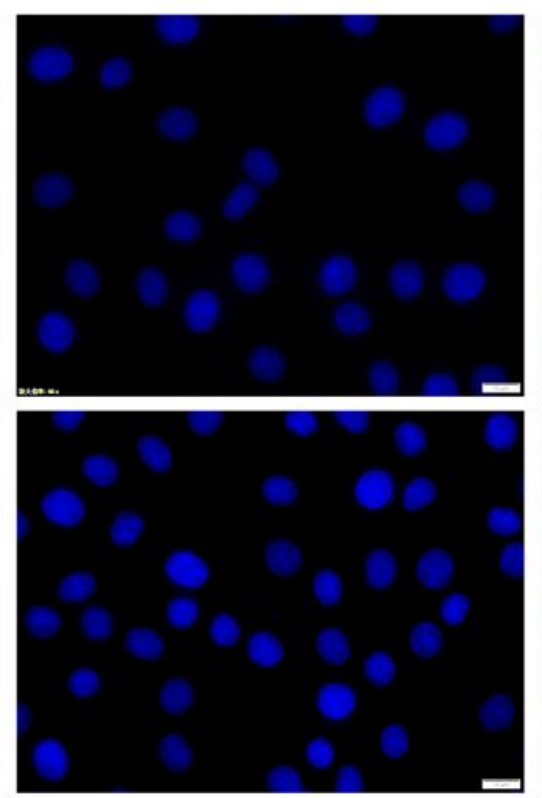

Merge
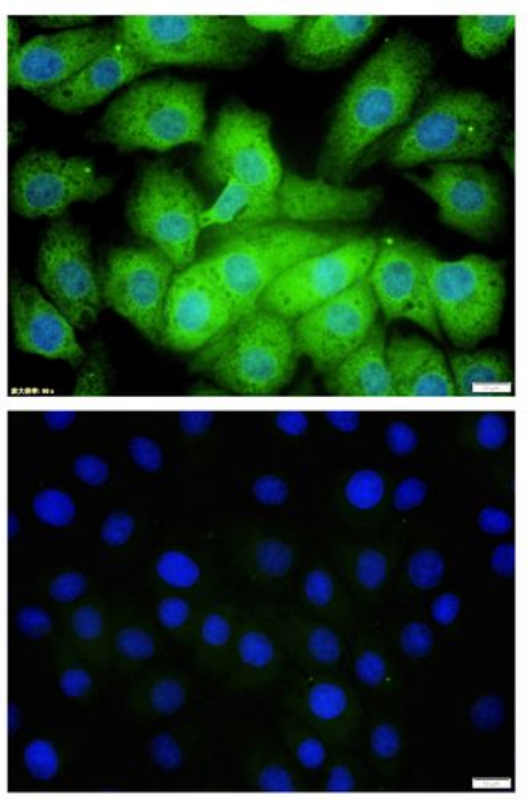

$\mathrm{C}$

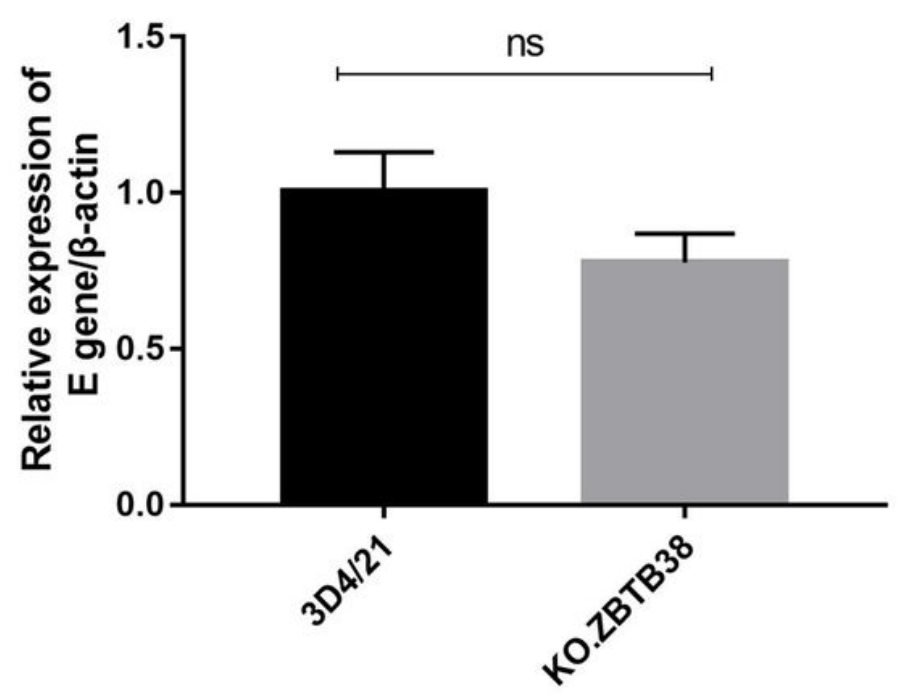

Figure 2

Identification of knockout cells and viral attachment and entry assay. A The indirect immunofluorescence assay was performed by using anti-ZBTB38 antibody to detect ZBTB38 protein (green) in KO.ZBTB38 cells (3D4/21 cells as control). DAPI (blue) for nuclear staining. The length of scale bars is $10 \mu \mathrm{m}$. Quantitative real-time RT-PCR was performed to determine mRNA levels of JEV in KO.ZBTB38 cells 
(3D4/21 cells as control, $\beta$-actin was used as the internal control) for viral attachment B and entry C assay. ns: not significant

A

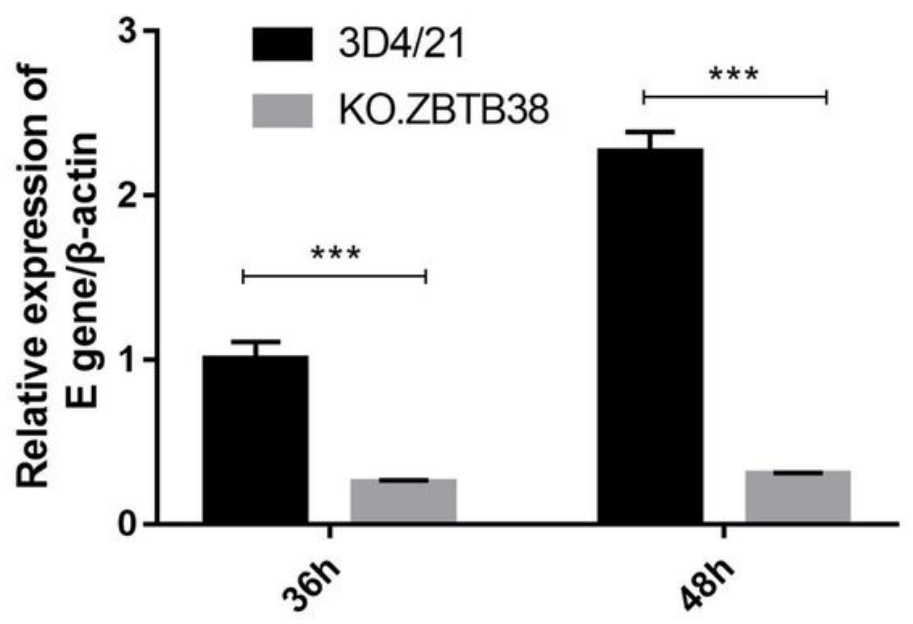

$\mathrm{B}$

$\mathrm{C}$
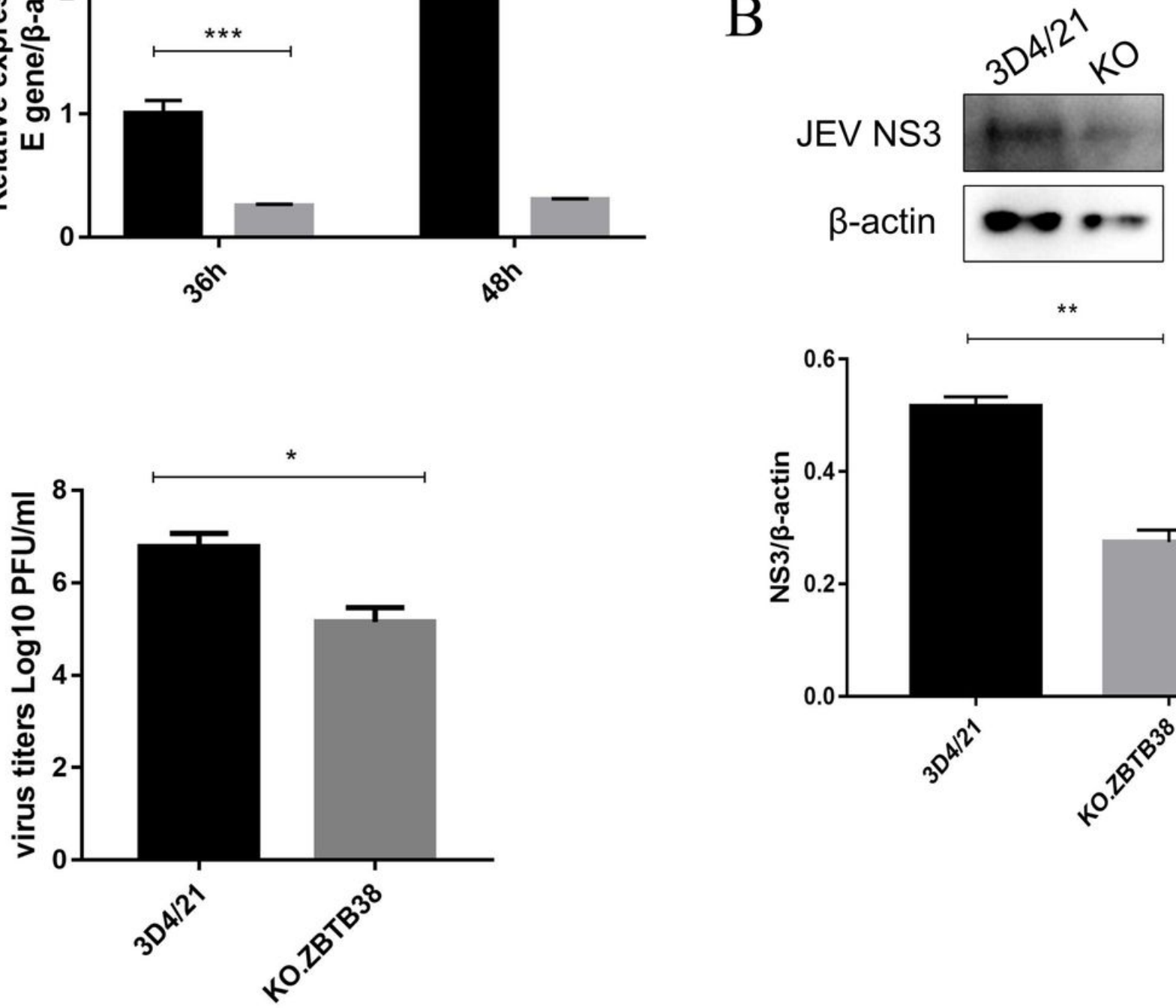

$\beta$-actin
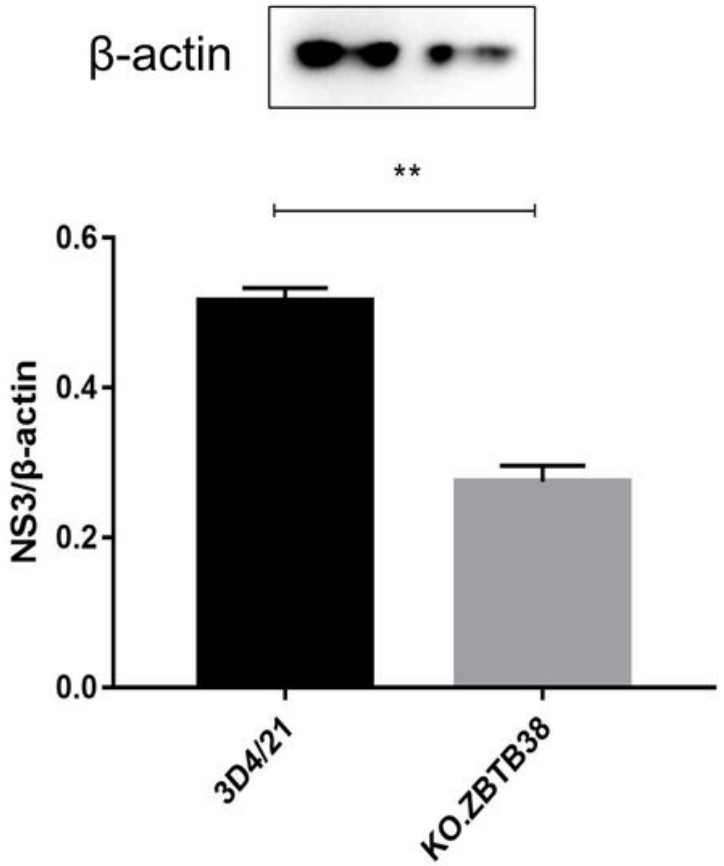

Figure 3

The effect of ZBTB38 deficiency on the replication and proliferation of JEV. A Quantitative real-time RTPCR for transcription level of JEV mRNA after ZBTB38 knockout cells were infected with JEV (MOI 1, 36 and $48 \mathrm{~h}$ post-infection) ( $\beta$-actin was used as the internal control). B Western blotting and grayscale quantitative analysis results revealed the expression level of NS3 protein after JEV infection (MOI 1, 48 $\mathrm{h}$ post-infection) in KO.ZBTB38 cells (3D4/21 cells as control). C KO.ZBTB38 cells were infected with JEV(MOI 1, $72 \mathrm{~h}$ post-infection) then plaque assay was perform to measure virus titer of JEV. ${ }^{\star * *} \mathrm{p}<$ $0.001,{ }^{* *} p<0.01,{ }^{*} p<0.05$ 


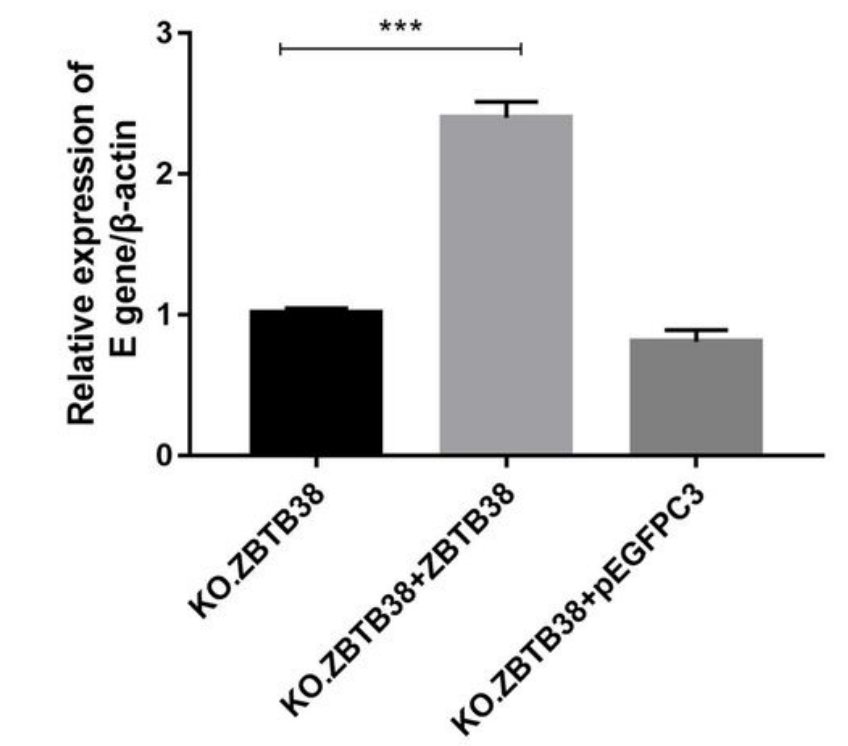

B

$\mathrm{C}$
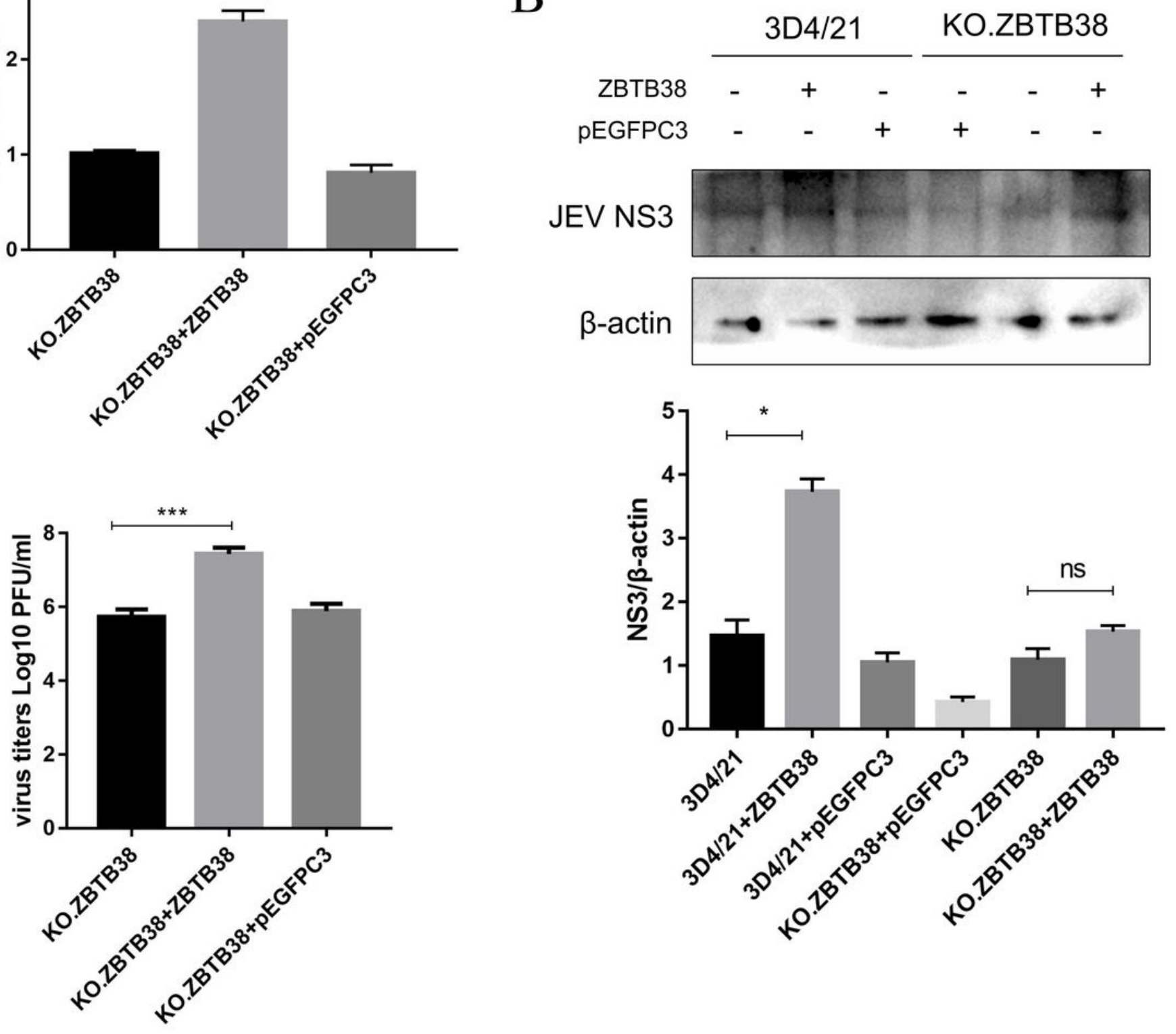

Figure 4

The effect of ZBTB38 overexpression on the replication and proliferation of JEV. The ZBTB38 recombinant plasmids were transfected into KO.ZBTB38 cells and 3D4/21 cells (negative control: transfected no plasmid, vector control: transfected empty vector), then cells were infected with JEV (MOI 1) and harvested for $48 \mathrm{~h}$ after infection. A Quantitative real-time RT-PCR for transcription level of JEV mRNA after ZBTB38 trans-complementing cells were infected ( $\beta$-actin was used as the internal control). B Western blotting and grayscale quantitative analysis results revealed the expression level of NS3 protein after JEV infection in ZBTB38 trans-complementing and overexpression cells. Cells were infected with JEV (MOI 1) and harvested for $72 \mathrm{~h}$ after infection. C Virus titer of JEV was measured by using plaque assay after trans-complementing ZBTB38 in KO.ZBTB38 cells. ${ }^{* \star *} p<0.001,{ }^{*} p<0.05$ 